\title{
En la senda de la extinción: el caso del algarrobo Prosopis chilensis (Fabaceae) y el bosque espinoso en la Región Metropolitana de Chile central
}

\section{In the path of extinction: the case of the mesquite Prosopis chilensis (Fabaceae) and the spiny forest at the Metropolitan Region of Central Chile}

\author{
Carlos E. Valdivia ${ }^{1 *}$ \& Cristian R. Romero² \\ ${ }^{1}$ Departamento de Ciencias Biológicas y Biodiversidad, Universidad de Los Lagos, Casilla 933, Osorno, Chile. \\ ${ }^{2}$ CSW, Consultores Ambientales, Camilo Henríquez 145, Depto. 304, Santiago, Chile. \\ *carlos.valdivia@ulagos.cl
}

\begin{abstract}
RESUMEN
En la zona central de Chile se encuentra el bosque espinoso de Acacia caven y Prosopis chilensis, donde persiste menos del $40 \%$ de su superficie original, aunque sin representación en las áreas silvestres protegidas. Debido a la acción del ganado, se ha sugerido una contracción poblacional de $P$. chilensis acoplado a una expansión poblacional de A. caven lo cual, sin embargo, no ha sido verificado empíricamente. En cuatro sitios correspondientes a dos microcuencas de la Región Metropolitana en Chile (Batuco y Huechún), se evaluó la incidencia de las perturbaciones ambientales (ganado, conejos, malezas, basuras), el estado fitosanitario y estructura poblacional de $P$. chilensis, así como las dinámicas de regeneración de este árbol y A. caven, mediante el uso de matrices de transiciones Markovianas. Las poblaciones de $P$. chilensis enfrentan similares grados de amenaza, exhiben un estado fitosanitario similar y deficitario y, consecuentemente, se encuentran en estado senescente prontas a desaparecer una vez muertos los individuos adultos de las siguientes dos generaciones. Además, la contracción poblacional de $P$. chilensis no se correlaciona con una expansión poblacional de $A$. caven, por lo cual las dos especies que definen y caracterizan el bosque espinoso de la Región Metropolitana de Chile se encuentran prontas a extinguirse y con ellas el piso vegetacional. Surge, por lo tanto, como necesidad urgente el establecer las medidas de conservación y restauración necesarias para asegurar la persistencia del bosque espinoso en la Región Metropolitana de Chile.
\end{abstract}

Palabras Clave: Algarrobos, espinos, degradación ambiental, extirpaciones futuras.

\begin{abstract}
At central Chile grows the spiny forest of Acacia caven and Prosopis chilensis of which less than $40 \%$ of its original surface currently persists, but with no representation into the wilderness protected areas. Owing to the action of cattle, has it been previously suggested a population reduction of $P$. chilensis coupled to a population increase of $A$. caven which, nonetheless, has not yet verified empirically. At four sites belonging to two micro-basins in the Metropolitan Region at central Chile (Batuco and Huechún), we assessed the incidence of environmental disturbances (cattle, rabbits, weeds, garbage), the phytosanitary stage, and population structure of $P$. chilensis, as well as the regeneration dynamics of this tree and $A$. caven by using Markovian transition matrices. Populations of $P$. chilensis suffer similar degrees of threats, exhibit a similar but depauperate phytosanitary stage and, consequently, they face a senescent stage prone to disappear when the current adult individuals go death. Furthermore, the population decrease of $P$. chilensis does not correlate with the previously suggested population increase of $A$. caven; hence, the tree species characterizing and defining the spiny forest of central Chile are prone to be extinct and with them the vegetation belt. It appears, therefore, as an urgent necessity the establishment of conservation and restoration programs in order to assure the persistence of the spiny forest at the Metropolitan Region in central Chile.
\end{abstract}

KEYwORDs: Mesquites, espinos, environmental degradation, future extirpations. 


\section{INTRODUCCIÓN}

La zona central de Chile presenta una flora rica en especies, endemismos y asociaciones vegetacionales (Arroyo et al. 1999). Sin embargo, la elevada concentración humana y los extensivos usos de la tierra han causado que muchas especies y tipos vegetacionales propios de esta zona se encuentren fuertemente amenazados, por lo cual esta región es considerada dentro de los sitios con prioridad mundial para la conservación de la biodiversidad (Arroyo et al. 1999, Myers et al. 2000).

Entre las unidades vegetacionales más amenazadas se encuentra el Bosque Espinoso Mediterráneo Interior de Acacia caven (Molina) Molina y Prosopis chilensis (Molina) Stuntz emend. Burkart (Luebert \& Pliscoff 2006). Este es un bosque abierto dominado por las especies ya mencionadas (algarrobo y espino, respectivamente), que se distribuye en los sectores planos o con pendiente suave de la depresión intermedia de las regiones de Valparaíso, Metropolitana y O’Higgins (Looser 1962, Luebert \& Pliscoff 2006). Si bien se ha sugerido que este bosque corresponde a una fase de degradación del bosque esclerófilo (Oberdorfer 1960), actualmente esto resulta improbable considerando las condiciones bioclimáticas en que se desarrolla (sensu Luebert \& Pliscoff 2006). Originalmente este bosque cubrió una superficie de $3.425 \mathrm{~km}^{2}$, de los cuales sólo persiste el $38,4 \%\left(1.316 \mathrm{~km}^{2}\right)$, aunque sin representación dentro de las unidades del Sistema Nacional de Áreas Silvestres Protegidas del Estado, SNASPE (Luebert \& Pliscoff 2006).

La destrucción de los espinos y particularmente del algarrobo en el bosque espinoso comenzó con la penetración española, durante la conquista y colonización, por el norte de Chile con un fuerte y creciente consumo con fines dendroenergéticos (Cunill 1971). De hecho, numerosos individuos de $P$. chilensis fueron talados, quemados o desprovistos de ramas provocando pérdidas directas e indirectas en el contingente poblacional debido a la destrucción total o parcial de los individuos (Looser 1962).

La depredación de semillas y plántulas por animales alóctonos silvestres (conejos) y domésticos (ganado bovino y caprino) también han contribuido a la degradación y reestructuración del bosque espinoso (Fuentes et al. 1989, Gutiérrez et al. 2007). De hecho, mientras las semillas de $A$. caven aumentan su capacidad germinativa tras pasar por el tracto digestivo del ganado, que actuaría como dispersor, $P$. chilensis disminuye significativamente su capacidad germinativa, por lo que el ganado actuaría como depredador (Fuentes et al. 1989). En el mismo estudio, Fuentes et al. (1989) determinaron que $P$. chilensis exhibe una población mayormente compuesta por adultos, indicador de una población en declinación, a diferencia de $A$. caven, que presenta un mayor contingente de juveniles, indicador de poblaciones en expansión (Fuentes et al. 1989). Por ello, se sugirió un proceso de transformación del bosque espinoso desde uno dominado por $P$. chilensis (i.e. algarrobal) hacia un matorral dominado por $A$. caven (i.e. espinal) mediado fundamentalmente por la acción del ganado bovino (Oberdorfer 1960, Looser 1962, Fuentes et al. 1989, Ovalle et al. 1993).

En síntesis, la degradación ambiental no sólo ha reducido el área de ocupación del piso de vegetación, sino también ha colocado en riesgo de extinción a una de las especies claves en la estructuración de este piso vegetacional, $P$. chilensis, considerada vulnerable por la legislación chilena (Benoit 1989).

El objetivo de este trabajo es evaluar la estructura y dinámica de reclutamiento de $P$. chilensis en cuatro sitios distintos de la Región Metropolitana, junto con la incidencia de los factores de degradación ambiental y fitosanitaria. Si las condiciones de degradación del bosque persisten, esperamos detectar futuras extinciones locales de las poblaciones de $P$. chilensis. Esperamos, además, que estas extinciones ocurran antes en aquellos sitios con una alta incidencia de los factores de degradación y, por lo tanto, que albergan poblaciones con una estructura etaria sesgada hacia individuos adultos más que juveniles. Finalmente, esperamos observar un reemplazo de los algarrobales por espinales producto de la actividad ganadera, tal como lo sugirió Fuentes et al. (1989) más de veinte años atrás.

\section{MÉTODOS}

Sitios y ESPECIES EN ESTUdiO

El trabajo de campo se realizó entre septiembre y octubre de 2009 en cuatro sitios de la zona norte de la Región Metropolitana, dos en la microcuenca de la laguna Batuco (sitio norte: 33¹2'53"S, 7049'26"W, 489 ms.n.m., sitio sur: $33^{\circ} 11^{\prime} 49^{\prime} \mathrm{S}, 70^{\circ} 50^{\prime} 04^{\prime \prime} \mathrm{W}, 488 \mathrm{ms.n.m}$. aprox.) $\mathrm{y}$ dos en la microcuenca del embalse Huechún (sitio norte: $33^{\circ} 03$ ' $22^{\prime}$ S, $70^{\circ} 47^{\prime} 58^{\prime \prime} \mathrm{W}, 586$ ms.n.m., sitio sur: 3306'48"S, 7048'54"W, 560 ms.n.m. aprox.) (Fig. 1). Ambas cuencas presentan suelos francos a franco-arcillolimosos en sus horizontes superficiales, y desde francoarcillo-limosos hasta arcillo-limosos en sus horizontes más profundos, los que se desarrollan sobre rocas del tipo andesitas y dioritas (véase Leighton 2010 para detalles). Ambas cuencas, además, se encuentran insertas dentro del bioclima Mediterráneo xérico-oceánico, en el cual se desarrolla el piso de vegetación bosque espinoso de $A$. caven y $P$. chilensis que se caracteriza por presentar una vegetación abierta, compuesta por especies esclerófilas como Cestrum parqui L'Hér., entre otras (véase Luebert \& Pliscoff 2006 para detalles). Sin embargo, en ambas cuencas destaca también la extendida presencia de actividades agropecuarias propias del secano interior de Chile y la consecuentemente escasa cobertura de vegetación nativa y elevada cobertura de vegetación alóctona. 


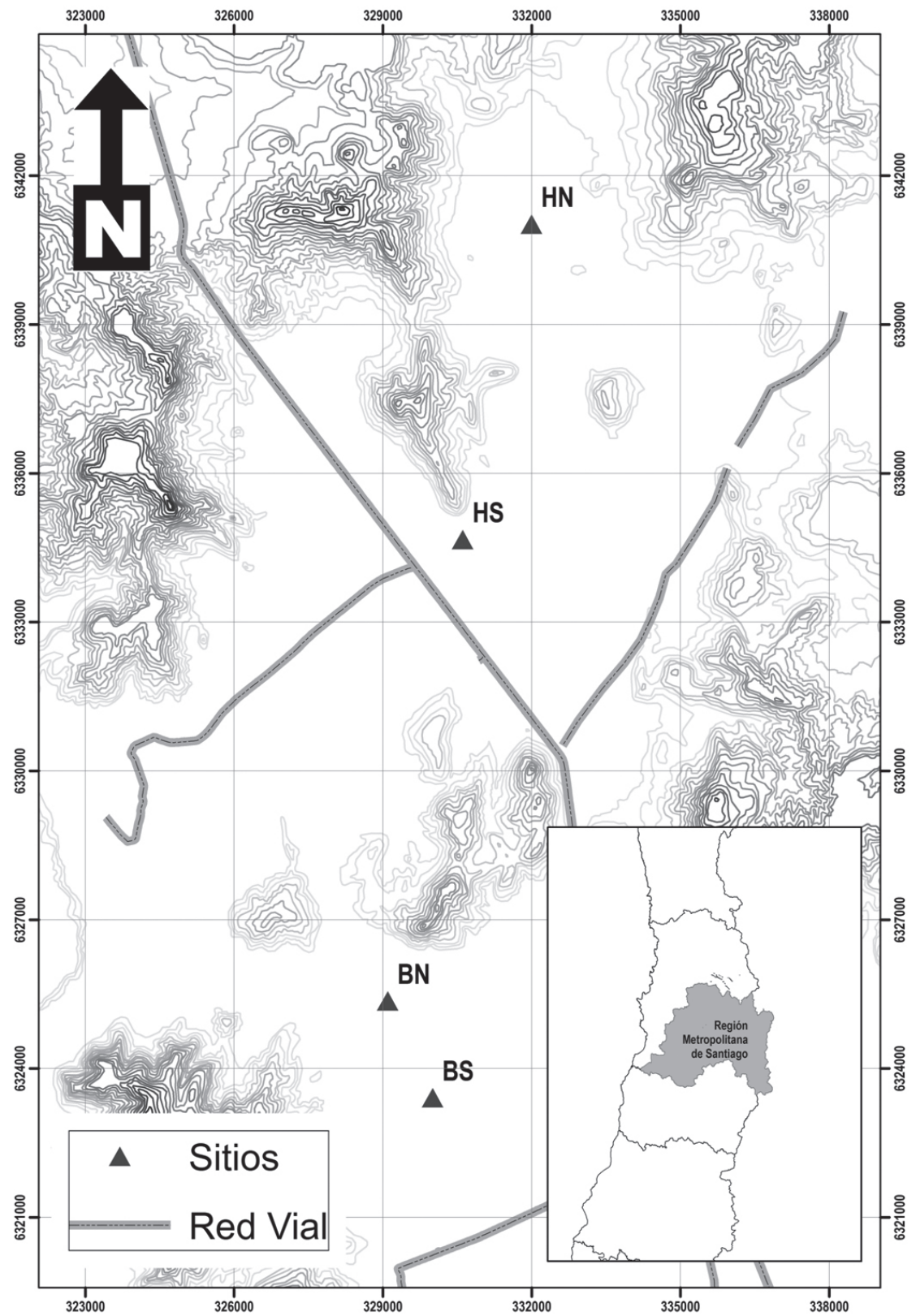

Figura 1. Localización de los sitios de estudio (UTM) de Prosopis chilensis y el bosque espinoso en la Región Metropolitana, Chile central. HN: Huechún sitio norte, HS: Huechún sitio sur, BN: Batuco sitio norte, BS: Batuco sitio sur. Cada línea de contorno indica un cambio de $75 \mathrm{~m}$ en elevación.

Figure 1. Study site location (UTM) of Prosopis chilensis and the spiny forest at the Metropolitan Region in central Chile. HN: Huechún sitio norte, HS: Huechún sitio sur, BN: Batuco sitio norte, BS: Batuco sitio sur. Each contour line indicates a $75 \mathrm{~m}$ change in elevation.

Prosopis chilensis es un árbol de hasta $10 \mathrm{~m}$ de altura con copa esférica y tronco de hasta $80 \mathrm{~cm}$ de diámetro a la altura del pecho (DAP) (Rodríguez et al. 1983). Presenta ramas flexibles y arqueadas con espinas de hasta $6 \mathrm{~cm}$ de longitud (Rodríguez et al. 1983). En sus ramas se desarrollan en primavera numerosas flores amarillas las cuales, al final del periodo reproductivo, dan origen a frutos indehiscentes del tipo legumbre de hasta $18 \mathrm{~cm}$ de largo con numerosas semillas de hasta 7,5 cm de longitud (Rodríguez et al. 1983). Aun cuando el piso vegetacional que estructura esta especie se distribuye entre las regiones de Valparaíso y O’Higgins, la distribución en Chile de $P$. chilensis es más extensa por el límite norte al crecer como especie azonal en las regiones de Atacama y Coquimbo (Rodríguez et al. 
1983). Esta especie está presente también en Perú, Bolivia y el noroeste de Argentina (Rodríguez et al. 1983).

Acacia caven es un árbol pequeño de hasta $6 \mathrm{~m}$ de altura con copa semiesférica y hasta $50 \mathrm{~cm}$ de DAP (Rodríguez et al. 1983). Presenta ramas gruesas, tortuosas, con numerosas espinas de hasta 2,5 cm de largo (Rodríguez et al. 1983). Durante la primavera desarrolla numerosas flores amarillas, que son polinizadas por insectos, las cuales posteriormente dan origen a frutos del tipo vaina leñosa de hasta $7 \mathrm{~cm}$ de largo con semillas de hasta $2,5 \mathrm{~cm}$ de longitud (Rodríguez et al. 1983). Su distribución geográfica en Chile es más amplia que en el caso de $P$. chilensis, creciendo entre las regiones de Atacama y Biobío (Rodríguez et al. 1983). Esta especie está presente también en Argentina, Brasil, Uruguay y Paraguay (Rodríguez et al. 1983).

Fuentes de PERTURbación y ESTAdo FItosanitario de PROSOPIS CHILENSIS

Para evaluar las fuentes de perturbación a las poblaciones de P. chilensis en cada sitio de muestreo se realizó un transecto de $1 \mathrm{~km}$ de largo y $20 \mathrm{~m}$ de ancho aprox., muestreando un total de ocho hectáreas. Dentro de cada transecto se dispusó 10 parcelas de $100 \mathrm{~m}^{2}(10 \times 10 \mathrm{~m})$ cada $100 \mathrm{~m}$ lineales, al interior de las cuales se registrá la presencia 0 ausencia de ganado y conejos, directa o indirectamente, a través de huellas, pelos o fecas, mediante simple inspección visual. Simultáneamente, para estimar la magnitud de la degradación ambiental se registrá también la presencia o ausencia de malezas (sensu Matthei 1995), así como basuras y escombros depositados clandestinamente.

Para evaluar el estado fitosanitario de las poblaciones de $P$. chilensis, en cada individuo vivo detectado al interior de cada transecto se registró si el árbol fue talado (i.e. pérdida de dominancia apical por corte del fuste), podado (i.e. pérdida de ramas por corte), quemado total o parcialmente, o bien se encontraba sin daño aparente.

Para evaluar si la frecuencia de las distintas fuentes de perturbaciones y los diferentes estados fitosanitarios de $P$. chilensis difieren significativamente entre los sitios y microcuencas, se realizaron pruebas exactas de Fisher para cada variable utilizando tablas de contingencia de $2 \times 2$ mediante el paquete estadístico Statistica versión 7.0.

\section{Estructura demográfica de PROSOPIS CHILENSIS}

Para estimar la estructura demográfica de $P$. chilensis, al interior de cada transecto de $20.000 \mathrm{~m}^{2}(\mathrm{n}=4)$ se registró la presencia de cada individuo de esta especie clasificándolos operacionalmente como plántula, cuando presentaron una altura menor de $50 \mathrm{~cm}$, juvenil, cuando presentaron alturas mayores a $50 \mathrm{~cm}$ y DAP menores de $10 \mathrm{~cm}$, y finalmente adulto cuando los individuos presentaron DAP $>10 \mathrm{~cm}$. En aquellos individuos con presencia de numerosas ramas a la altura del pecho debido a la pérdida de dominancia apical, el DAP se estimó por la sumatoria de los diámetros de cada rama (sensu Álvarez et al. 2006). Para evaluar si el número de individuos totales y en cada estado etario difiere significativamente entre los sitios y microcuencas, se realizaron pruebas exactas de Fisher para cada variable utilizando tablas de contingencia de $2 \times 2$ mediante el paquete estadístico Statistica versión 7.0.

\section{Densidades poblacionales futuras de Prosopis Chilensis Y} ACACIA CAVEN

Para estimar los tamaños poblacionales futuros de $P$. chilensis y un posible reemplazo de esta especie por $A$. caven, al interior de cada parcela de $100 \mathrm{~m}^{2}$ se registrarón todos los individuos adultos y la identidad y número de cada individuo juvenil o plántula de ambas especies creciendo bajo el dosel de los árboles adultos. Posteriormente, para cada sitio se elaboró una matriz de transiciones Markovianas donde se determinó la probabilidad que los árboles adultos tienen de ser reemplazados por juveniles o plántulas con- o hetero-específicas (Facelli \& Pickett 1990). Las probabilidades de transición $(P)$ se obtuvieron mediante el cociente entre el número total de reclutas (plántulas más juveniles de una determinada especie) creciendo bajo el dosel de los adultos $(A)$ de una determinada especie. El vector de estado actual $\left(v_{0}\right)$ correspondió al número total de árboles adultos observados de cada especie. El modelo se parametrizó como sigue:

$$
\left[\begin{array}{ll}
P i i & P i j \\
P j i & P j j
\end{array}\right] \times\left[\begin{array}{l}
A i \\
A j
\end{array}\right]
$$

donde $i$ representa a $A$. caven y $j$ representa a $P$. chilensis. La matriz de transiciones se iteró por los vectores de estado $\left(v_{\mathrm{n}}\right)$ resultantes de cada ponderación, hasta alcanzar la fase de equilibrio (i.e. $\mathrm{n}=4$ iteraciones o recambios generacionales). En el presente modelo asumimos que cada especie en estado adulto podría ser reemplazada por un número variable de individuos, de la misma u otra especie, a los actualmente observados, dando cabida a hipotéticos incrementos o disminuciones poblacionales $\mathrm{y}$, consecuentemente, a variaciones en las densidades arbóreas. Matemáticamente, esto se observa en el modo en que se estimaron las probabilidades de transición de la matriz, las cuales pueden adoptar valores putativos $>1$ en cada celda. La formulación de este modelo, con variaciones en el número total de individuos en la comunidad forestal, se basó en la reducción histórica de la densidad de árboles en este piso de vegetación (Fuentes et al. 1989), lo cual nos sugiere que los sitios en estudio tienen la capacidad de albergar tamaños y densidades poblacionales mayores, de ambas especies, a los actualmente observados.

Considerando que la presencia de especies arbóreas distintas a $A$. caven y $P$. chilensis en los sitios de estudio puede modificar las matrices de transiciones antes señaladas (Facelli \& Pickett 1990), se verificó la presencia de adultos o 
plántulas de otras especies arbóreas creciendo bajo el dosel de las especies focales, así como la presencia de juveniles o plántulas de las especies focales creciendo bajo el dosel de otras especies arbóreas. Sólo se observó la presencia de Porlieria chilensis I.M.Johnst. (guayacán) creciendo en el sitio norte de Batuco como especie acompañante. Sin embargo, dado que ésta no exhibió regeneración bajo el dosel de las especies focales y, a su vez, A. caven y $P$. chilensis no regeneraron bajo Porlieria chilensis, podemos descartar cualquier efecto de esta última especie sobre la matriz de transiciones.

Adicionalmente, dado que el presente modelo no permitiría estimar con certeza la regeneración de las especies focales si existiesen individuos regenerando en sitios abiertos, se verificó también la presencia de plántulas o juveniles fuera del dosel arbóreo, pero al interior de cada parcela. Al respecto, no se detectarón reclutas creciendo en sitios abiertos.

\section{RESULTADOS}

Fuentes de Perturbación y estado fitosanitario de PRosopis CHILENSIS

La frecuencia en la incidencia de ganado, conejos, malezas y basuras-escombros en cada población estudiada de $P$. chilensis no se asoció significativamente a que éstos crezcan en un determinado sitio o microcuenca (Prueba exacta de Fisher de dos colas: $P=0,127 ; P=0,282 ; P=1,000 ; P=$ 0,308; respectivamente) (Tabla I).

Similarmente, el número de individuos de $P$. chilensis talados, podados o quemados en cada población estudiada no se asoció significativamente a que éstos crezcan en un determinado sitio o microcuenca (Prueba exacta de Fisher de dos colas: $P=0,619 ; P=0,069 ; P=0,333$; respectivamente) (Tabla II).
Estructura demográfica de PROSOPIS CHILENSIS

El número de juveniles, adultos, así como el número total de individuos de $P$. chilensis en cada población estudiada no se asoció significativamente a que éstos crezcan en un determinado sitio o microcuenca (Prueba exacta de Fisher de dos colas: $P=1,000 ; P=0,619 ; P=0,627$; respectivamente) (Fig. 2). Además, en los cuatro sitios estudiados se observaron más individuos adultos que juveniles, y en el caso de los sitios en Huechún, más juveniles que plántulas. De hecho, en Batuco sur no se registraron individuos en estado de plántula, mientras que en Batuco norte tampoco se registraron juveniles (Fig. 2).

Densidades poblacionales futuras de Prosopis Chilensis Y ACACIA CAVEN

En Batuco norte, el número de adultos de $P$. chilensis fue 16 veces mayor que el de juveniles (16 adultos / $1000 \mathrm{~m}^{2}$ y 1 juvenil / $1000 \mathrm{~m}^{2}$ ), mientras que el número de adultos de $A$. caven fue 5 veces mayor (5 adultos / $1000 \mathrm{~m}^{2}$ y 1 juvenil / $1000 \mathrm{~m}^{2}$ ). En Batuco sur, el número de adultos de $P$. chilensis fue 5 veces mayor que el de juveniles (5 adultos / $1000 \mathrm{~m}^{2}$ y 1 juvenil / $1000 \mathrm{~m}^{2}$ ), mientras que el número de adultos de $A$. caven fue 15 veces mayor (15 adultos / 1000 $\mathrm{m}^{2}$ y 1 juvenil / $1000 \mathrm{~m}^{2}$ ). Similarmente, en Huechún norte, el número de adultos de $P$. chilensis fue 3 veces mayor que el de juveniles ( 6 adultos / $1000 \mathrm{~m}^{2}$ y 3 juveniles / 1000 $\mathrm{m}^{2}$ ), mientras que el número de adultos de $A$. caven fue de 24, no registrándose juveniles. En Batuco sur, sólo se registró 6 individuos adultos de $P$. chilensis, mientras que el número de adultos de $A$. caven fue 6,2 veces mayor que el de juveniles ( 37 adultos / $1000 \mathrm{~m}^{2}$ y 6 juveniles / $1000 \mathrm{~m}^{2}$ ).

Consecuentemente, debido al menor contingente de juveniles que de adultos de ambas especies en los cuatro sitios en estudio, la densidad de individuos de ambos árboles se reducirá progresivamente en las próximas generaciones hasta la extinción poblacional (Fig. 3).

TABLA I. Frecuencia de las fuentes de perturbación e indicadores de degradación ambiental observados en el bosque espinoso de Prosopis chilensis y Acacia caven en Chile central.

TABLE I. Frequency of disturbance sources and indicators of environmental degradation observed at the spiny forest of Prosopis chilensis and Acacia caven in central Chile.

\begin{tabular}{lrrrr}
\hline \multirow{2}{*}{ Fuente de perturbación } & \multicolumn{2}{c}{ Batuco } & \multicolumn{2}{c}{ Huechún } \\
\cline { 2 - 5 } & Sitio Norte & Sitio Sur & Sitio Norte & \multicolumn{1}{c}{ Sitio Sur } \\
\hline Ganado & $10(100 \%)$ & $5(50 \%)$ & $1(10 \%)$ & $4(40 \%)$ \\
Conejos & $4(40 \%)$ & $4(40 \%)$ & $1(10 \%)$ & $7(70 \%)$ \\
Malezas & $10(100 \%)$ & $10(100 \%)$ & $10(100 \%)$ & $10(100 \%)$ \\
Basura y escombros & $1(10 \%)$ & $3(30 \%)$ & $0(0 \%)$ & $9(90 \%)$ \\
N $^{\circ}$ de parcelas / sitio & 10 & 10 & 10 & 10 \\
\hline
\end{tabular}


Tabla II. Estado fitosanitario de Prosopis chilensis evaluado como la frecuencia de individuos con daño por tala, poda y/o fuego. El número total de individuos para cada sitio no corresponde a la suma de acuerdo al estado fitosanitario debido a que algunos individuos presentaron más de un daño a la vez.

TABLE II. Phytosanitary stage of Prosopis chilensis assessed as the frequency of individuals with damage triggered by logging, pruning, and/or fire. The number of total individuals per site does not correspond to the sum of each one within each phytosanitary stage because some individuals exhibited more than one type of damage simultaneously.

\begin{tabular}{lcccc}
\hline \multirow{2}{*}{ Estado sanitario } & \multicolumn{2}{c}{ Batuco } & \multicolumn{2}{c}{ Huechún } \\
\cline { 2 - 5 } & Sitio Norte & Sitio Sur & Sitio Norte & Sitio Sur \\
\hline Talados & $2(11,8 \%)$ & $3(8,8 \%)$ & $18(23,7 \%)$ & $47(38,2 \%)$ \\
Podados & $2(11,8 \%)$ & $7(20,6 \%)$ & $20(26,3 \%)$ & $14(11,4 \%)$ \\
Quemados & $2(11,8 \%)$ & $0(0,0 \%)$ & $0(0,0 \%)$ & $1(0,8 \%)$ \\
$\mathrm{N}^{\circ}$ de individuos / sitio & 17 & 34 & 76 & 123 \\
\hline
\end{tabular}

Batuco

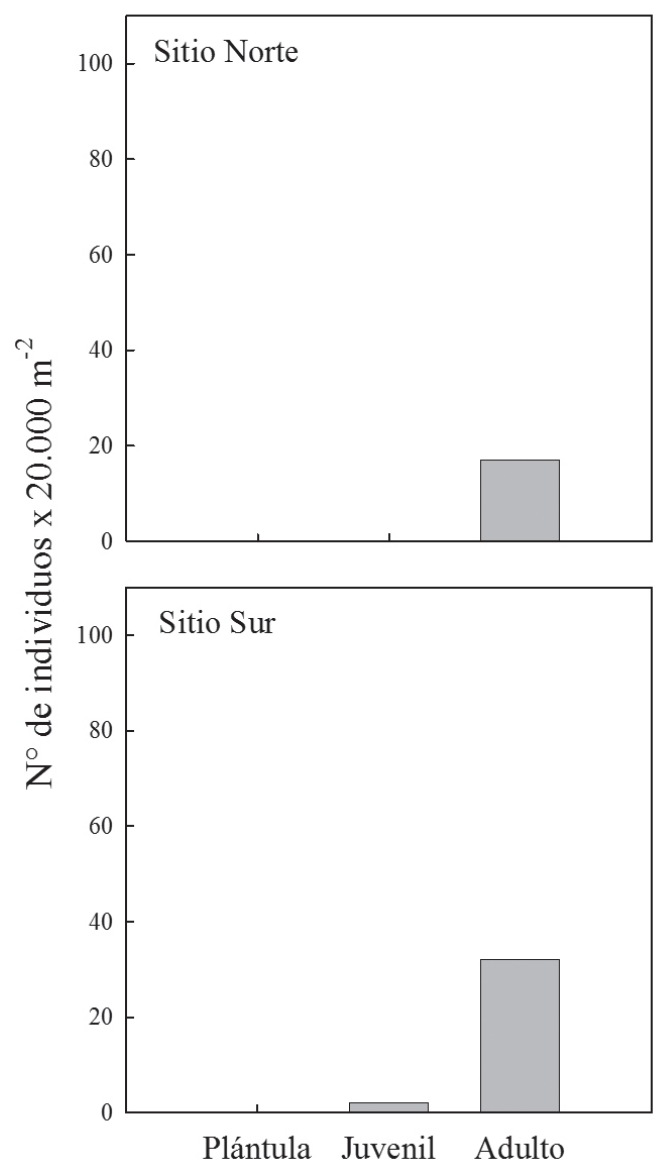

Huechún
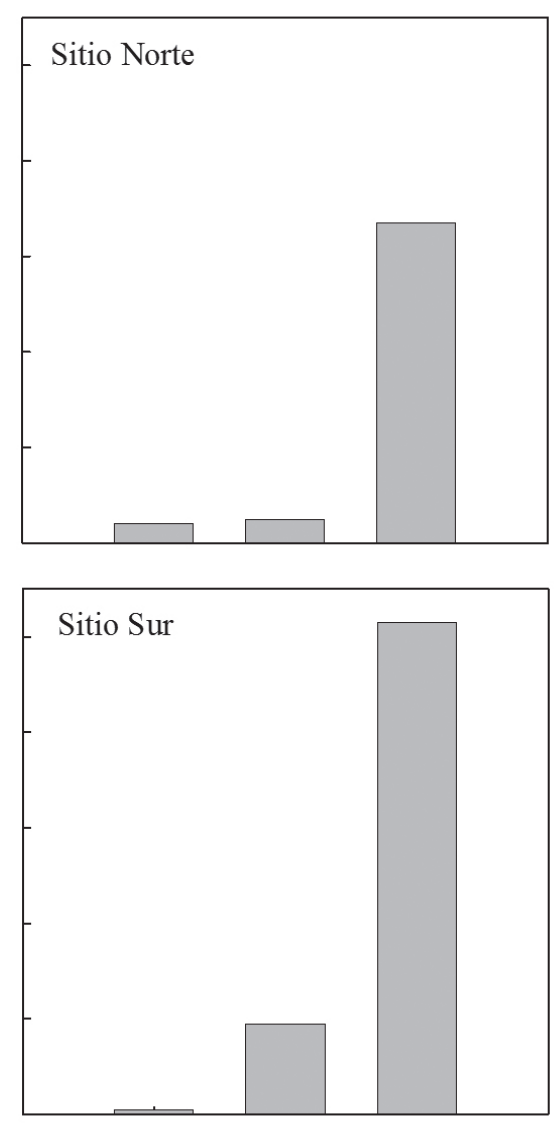

Plántula Juvenil Adulto

FIgURA 2. Estructura poblacional de Prosopis chilensis en cuatro sitios correspondientes a dos microcuencas en Chile central.

FIgURE 2. Population structure of Prosopis chilensis at four sites belonging to two micro-basins in central Chile. 
Batuco

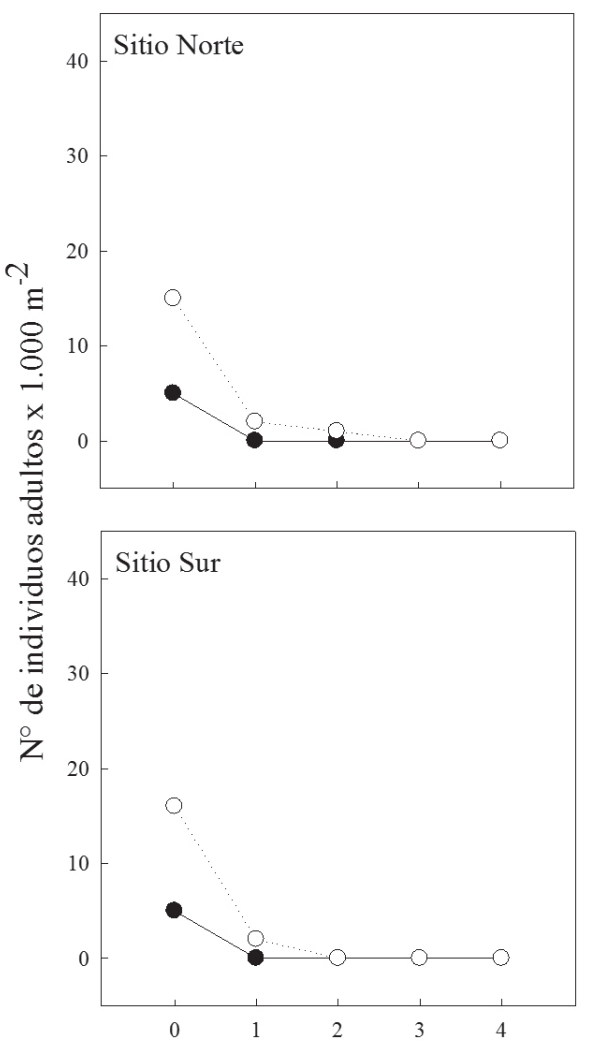

Huechún
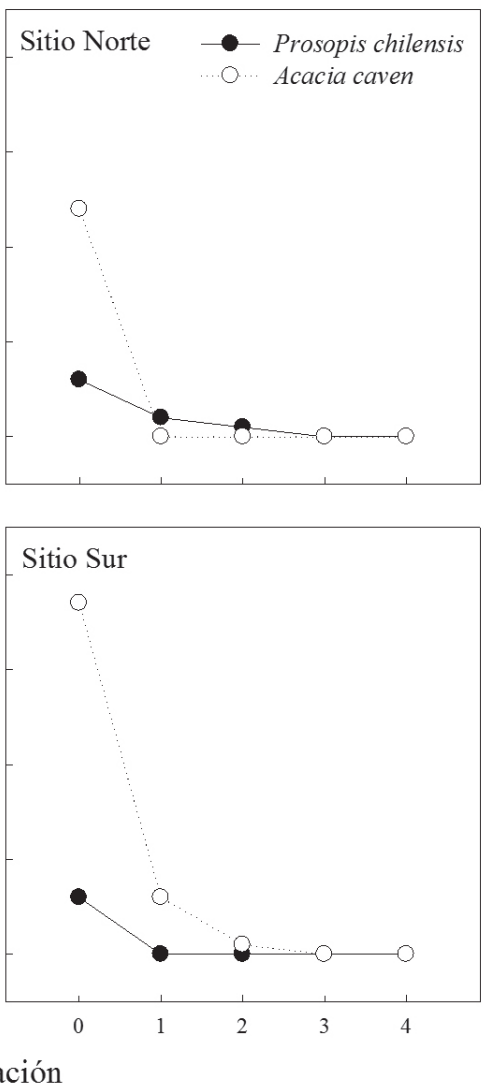

Figura 3. Densidad poblacional observada (generación 0) y estimada mediante matrices de transiciones Markovianas (generación 1,..., 4) de Prosopis chilensis y Acacia caven en cuatro sitios correspondientes a dos microcuencas en Chile central, una vez transcurridos cuatro recambios generacionales.

Figure 3. Population density observed (generation 0) and estimated through Markovian transition matrices (generation 1,..., 4) of Prosopis chilensis and Acacia caven at four sites belonging to two micro-basins in central Chile elapsing four generation turnovers.

\section{DISCUSIÓN}

Las poblaciones de $P$. chilensis enfrentan similares grados de amenaza, exhiben un estado fitosanitario similar aunque deficitario y, consecuentemente, se encuentran en estado senescente prontas a desaparecer una vez muertos los individuos adultos de las siguientes dos generaciones. Desafortunadamente, no se conoce el tiempo exacto en que esto sucederá, dado el desconocimiento de los tiempos necesarios para que ocurran los recambios generacionales en $P$. chilensis. Al respecto, es importante notar además que es sabido que las especies arbóreas de la zona centronorte de Chile exhiben pulsos de reclutamiento asociados a las fuertes precipitaciones que ocurren como consecuencia del fenómeno de El Niño (ENSO, por su sigla en inglés) cada siete años aproximadamente, por lo cual no es factible esperar estructuras demográficas con una mayor cantidad de plántulas que de adultos (Gutiérrez et al. 2007). Sin embargo, considerando que el presente modelo fue construido a partir de los estados juveniles de hasta diez años de edad aproximadamente (véase Fuentes et al. 1989), se puede señalar con confianza que la degradación ambiental de origen humano es la causa de la casi nula regeneración poblacional aquí documentada. Esto, por cierto, podría estar retroalimentándose con las variaciones interanuales naturales debido al fenómeno de El Niño previamente reportadas.

La contracción poblacional de $P$. chilensis que indica nuestro modelo no se correlaciona con la supuesta expansión poblacional de $A$. caven previamente sugerida por otros estudios, por lo cual y al menos en las microcuencas de Batuco y Huechún, el algarrobal no se transformaría en un espinal. De hecho, si se considera ambas microcuencas como sitios representativos del piso vegetacional, las dos especies que definen y caracterizan este tipo de bosque estarían prontas a extinguirse, desapareciendo con ellas el bosque espinoso de los valles centrales de Chile.

La presencia de escombros y basuras en los sitios en estudio indican que las poblaciones de $P$. chilensis y $A$. caven podrían enfrentar severas limitaciones en su reclutamiento 
debido al daño mecánico que putativamente experimentarían las plántulas simplemente al verse aplastadas por este tipo de materiales. La presencia de malezas podría significar una amenaza adicional al reclutamiento de los árboles aquí estudiados debido a la competencia por los sitios de reclutamiento; mientras que la presencia de conejos y ganado contribuiría a dicha limitación del reclutamiento al depredar sobre las plántulas de éstos árboles. Desafortunadamente, todos estos factores se encuentran presentes simultánea y similarmente en los cuatro sitios aquí evaluados, dando pocas oportunidades a las plántulas de establecerse con éxito. Esta situación resultaría aún más perjudicial si se considera que el reclutamiento de este tipo de árboles en climas semiáridos está fuertemente limitado a la ocurrencia de años lluviosos (Gutiérrez et al. 2007).

Prosopis chilensis, junto con A. caven, tiene la facultad de fijar nitrógeno contribuyendo positivamente en la estructuración del suelo y al adecuado desarrollo de las plantas que allí crecen (Ovalle et al. 1999, Urzúa 2005). Por ello y debido a la histórica reducción en el contingente poblacional de esta especie es que actualmente los suelos donde se desarrolla el bosque espinoso se encuentran fuertemente compactados y con escasa actividad microbiana, provocando una pobre incorporación de micronutrientes $\mathrm{y}$, por lo tanto, restringiendo fuertemente la capacidad de reclutamiento de la especies que aquí crecen (Ovalle et al. 1999). Esta pérdida de nutrientes en el suelo, ciertamente, podría estar actuando sinérgicamente con los factores de degradación de las poblaciones de $P$. chilensis y A. caven aquí reportados, contribuyendo así a intensificar la reducción en el contingente poblacional que recluta año a año. Bajo este escenario, es indudable que la pérdida de $P$. chilensis en particular, y del bosque espinoso en general, tenga consecuencias negativas más allá de las meramente biológicas al afectar, por ejemplo, la economía de los pequeños agricultores que habitan en este piso de vegetación. De hecho, en esta zona habitan alrededor de 300.000 personas vinculadas directa o indirectamente a la actividad agropecuaria, la cual se ve mermada por la pérdida de calidad de los suelos (Ovalle et al. 1990, 1996, Aronson et al. 1998).

Prosopis chilensis exhibe adaptaciones fisiológicas que le permiten desarrollarse en suelos salinos y tolerar condiciones estresantes de déficit hídrico, daños mecánicos y drásticos cambios de temperatura (Felker et al. 1983, Arce et al. 1990, Medina \& Cardemil 1993, Rodríguez \& Cardemil 1994, 1995, Cazebonne et al. 1999). Por todo esto, $P$. chilensis podría contribuir sustancialmente a la recuperación, mantención y enriquecimiento de los suelos actualmente degradados (Ovalle et al. 1999). A esto se suma la potencialidad que tienen los frutos de esta especie de constituirse en una importante fuente de nutrientes para el ganado y las personas (Silva et al. 2000, Aedo 2007, Galán et al. 2008, Escobar et al. 2009). Por lo tanto, es ciertamente factible y necesario establecer estrategias de conservación y restauración que compatibilicen tanto propósitos conservacionistas como agro-productivos.

No obstante, aun cuando $P$. chilensis provee importantes bienes y servicios actuales y potenciales, desde el periodo colonial a la fecha ha habido una reducción significativa en los usos de dichos recursos, por lo cual, asociado al proceso de extinción biológica aquí reportado, ha existido un creciente proceso de extinción cultural en torno a esta especie y a la unidad vegetacional que integra. Ciertamente, la desvinculación cultural con este tipo de bosque, y particularmente con P. chilensis, ha sido el detonante del magro estado fitosanitario junto con la alta incidencia de los factores de degradación ambiental aquí reportados, lo cual contribuye a incrementar aun más el riesgo de extinción de esta especie y la comunidad biológica que estructura. Por lo tanto, resulta mandatorio que ambos fenómenos de extinciones, tanto biológica como cultural, sean revertidos en el corto plazo emprendiendo acciones de conservación y restauración como: i) designar áreas silvestres protegidas que incluyan este piso de vegetación, ii) establecer programas educativos para los asentamientos locales que enseñen sobre los beneficios y servicios aportados por $P$. chilensis, y iii) plantear programas de restauración ambiental y reforestación que contribuyan a enriquecer los suelos actualmente degradados por su corta indiscriminada para así incrementar el contingente poblacional y, de este modo, revertir la extinción inminente de esta especie arbórea y el bosque espinoso en la Región Metropolitana de Chile.

\section{AGRADECIMIENTOS}

Agradecemos los comentarios y sugerencias de dos árbitros anónimos de Gayana Botánica.

\section{REFERENCIAS}

Aedo, R.A. 2007. Factibilidad técnico-económica de generar productos alimenticios a partir del fruto del algarrobo chileno (Prosopis chilensis) para la alimentación humana o animal. Tesis de Licenciatura en Agronomía. Facultad de Ciencias Agrarias, Universidad Austral, Valdivia, Chile.

Álvarez, J.A., P.E. Villagra, M.A. Cony, E. Cesca \& J.A. Boninsegna. 2006. Estructura y estado de conservación de los bosques de Prosopis flexuosa DC. en el Noreste de Mendoza, Argentina. Revista Chilena de Historia Natural 79: 75-87.

Arce, P., C. Medina \& O. Balboa. 1990. Tolerancia a la salinidad en tres especies de Prosopis ( $P$. alba, P. chilensis y $P$. tamarugo). Ciencia e Investigación Agraria 17: 1-75.

Aronson, J., A. del Pozo, C. Ovalle, J. Avendaño, A. Lavin \& M. ETIENNE. 1998. Land use changes and conflicts in central Chile. In P.W. Rundel, G. Montenegro \& F. 
Jaksic (eds.), Landscape disturbance and biodiversity in Mediterranean-type ecosystems, p. 155-168. Springer, Berlin.

Arroyo, M.T.K., R. Rozzi, J.A. Simonetti, P. Marquet \& M. SAlaberry. 1999. Central Chile. In Mittermeier, R.A., N. Myers, P. Robles Gil \& C.G. Mittermeier (eds.), Hotspots: Earth's Biologically Richest and Most Endangered Terrestrial Ecorregions, p. 161-171. CEMEX, México.

Benoit, I. (ED.)1989. Red List of Chilean Terrestrial Flora. (Part One) CONAF, Santiago de Chile. 151 p.

Cazebonne, C., A.I. Vega, D.A. Varela \& L.A. Cardemil. 1999. Salinity effects on germination and growth of Prosopis chilensis. Revista Chilena de Historia Natural 72: 83-91.

CunILl, P. 1971. Factores de destrucción del paisaje chileno; recolecciones, caza y tala coloniales. Revista Informaciones Geográficas: $N^{\circ}$ 235-264.

Escobar, B., A.M. Estévez, C. Fuentes \& D. Venegas. 2009. Uso de harina de cotiledón de algarrobo (Prosopis chilensis) como fuente de proteína y fibra dietética en la elaboración de galletas y hojuelas fritas. Archivos Latinoamericanos de Nutrición 59: 191-198.

Facelli, J.M. \& S.T.A. Pickett. 1990. Markovian chains and the role of history in succession. Trends in Ecology and Evolution 5: 27-30.

Felker, P., G.H. Cannell, P.R. Clark, J.F. Osborn \& P. Nash. 1983. Biomass production of Prosopis species (mesquite), Leucaena, and other leguminous trees grown under heat drought stress. Forestry Science 29: 592-606.

Fuentes, E.R., R. Avilés \& A. Segura. 1989. Landscape change under indirect effects of human use: the Savanna of central Chile. Landscape Ecology 2: 73-80.

Galán, A.G., A.D. Correa, C.M.P. Abreu \& M.F.P. Barcelos. 2008. Caracterización química de la harina del fruto de Prosopis spp. procedente de Bolivia y Brasil. Archivos Latinoamericanos de Nutrición 58: 309-315.

Gutiérrez, J.R., M. Holmgren, M. Manrique \& F.A. Squeo. 2007. Reduced herbivore pressure under rainy ENSO conditions could facilitate dryland reforestation. Journal of Arid Environments 68: 322-330.

Leighton, W.L. 2010. Suelos de Chile. Impresos Maval, Santiago Chile. 364 pp.

Looser, G. 1962. La importancia del algarrobo (Prosopis chilensis) en la vegetación de la provincia de Santiago, Chile. Revista de la Universidad Católica de Chile 47: 103-116.

Luebert, F. \& P. Pliscoff. 2006. Sinopsis bioclimática y vegetacional de Chile. Editorial Universitaria, Santiago, Chile. 316 pp.

Matthei, O. 1995. Manual de las Malezas que Crecen en Chile. Alfabeta Impresores. Santiago, Chile. 545 pp.

Medina, C. \& L. Cardemil. 1993. Prosopis chilensis is a plant highly tolerant to heat shock. Plant Cell and Environment 16: 305-310.

Myers, M., R.A. Mittermeier, G.A.B. Fonseca \& J. Kent. 2000. Biodiversity hotspots for conservation priorities. Nature 40: 853-858.

Oberdorfer, E. 1960. Pflanzensoziologische studien in Chile. Ein Vergleich mit Europa. Flora et Vegetatio Mundi 2: 1-208.

Ovalle, C., J. Aronson, J. Avendaño, R. Meneses \& R. Moreno. 1993. Rehabilitation of degraded ecosystems in central Chile and its relevance to the arid "Norte chico". Revista Chilena de Historia Natural 66: 291-303.

Ovalle, C., J. Aronson, A. del Pozo \& J. Avendaño. 1990. The espinal: agroforestry systems of the Mediterranean-type climate región of Chile. Agroforestry Systems 10: 213239.

Ovalle, C., J. Aronson, A. del Pozo \& J. Avendaño. 1999. Restoration and rehabilitation of mixed espinales in central Chile: 10-years report and appraisal. Arid Soil Research and Rehabilitation 13: 369-381.

Ovalle, C., J. Avendaño, J. Aronson \& A. Del Pozo. 1996. Espinales of subhumid central Chile. I. Cartography of land occupation. Forest Ecology and Management 86: 129-139.

Rodríguez, J.G. \& L. CARdemil. 1994. Cell wall proteins in seedling cotyledons of Prosopis chilensis. Phytochemistry 35: 281-286.

Rodríguez, J.G. \& L. Cardemil. 1995. Tissue specific expression of cell wall proteins of seedlings of Prosopis chilensis during development and wound stress. Physiologia Plantarum 93: 457-463.

Rodríguez, R., O. Matthei \& M. Quezada. 1983. Flora Arbórea de Chile. Ediciones Universidad de Concepción, Alfabeta Impresos. Santiago, Chile. 408 pp.

Silva. M.P., M.J. Martínez, R. Corini, M.A. Brunetti, M. BALZARINI \& U. KARLIN. 2000. Valoración nutritiva del fruto del algarrobo (Prosopis chilensis) bajo distintos tipos de almacenamiento. Multequina 9: 65-74.

UrzÚA, H. 2005. Beneficios de la fijación simbiótica de nitrógeno en Chile. Ciencia e Investigación Agraria 32: 133-150.

Recibido: 03.05.12

Aceptado: 20.11.12 